\title{
Un Modelo de Prácticas para analizar el Proceso Social de Institucionalización Escolar del Conocimiento Matemático
}

\author{
A Practice Model to Analyze the Social Process of Academic \\ Institutionalization of Mathematical Knowledge
}

\begin{abstract}
Verónica Molfino Vigo*
Gabriela Buendía Ábalos**

Resumen

El proceso de transición de un saber matemático desde que es concebido y desarrollado en el ámbito científico hasta su difusión en las aulas ha sido estudiado por diversos autores y desde diferentes perspectivas de la Matemática Educativa. En este artículo se describe un marco teórico-metodológico que permite analizar ese proceso, que hemos denominado proceso social de institucionalización, poniendo el foco de atención en las prácticas de los actores involucrados en cada uno de sus momentos. El modelo se desarrolla bajo la perspectiva socioepistemológica y se apoya en la teoría del análisis del discurso como acción social para estudiar el discurso matemático escolar.En particular, se desarrolla la manera en que se ha empleado el marco teórico-metodológico para analizar el proceso social de institucionalización del concepto de límite en el contexto educativo uruguayo. Se explora, a su vez, su posible extensión al proceso de institucionalización que atraviesan otros saberes matemáticos, en otros contextos socioculturales.
\end{abstract}

Palabras clave: Proceso Social de Institucionalización.Conocimiento Matemático. Modelo teóricometodológico.

\begin{abstract}
The transition process of mathematical knowledge, since its conception and development by mathematicians to its diffusion in the classroom, has been studied by several authors and from different perspectives of Mathematics Education. This article describes a theoretical and methodological framework to analyze this process, which we call the social process of institutionalization, focusing on the practices of the actors involved in each of its moments. The model is developed from the socio-epistemological perspective and relies on the theory of discourse analysis as a social action to study school mathematical discourse. In particular, it is developed the way the theoretical and methodological framework was used to analyze the social process of institutionalization of the concept of limit on the Uruguayan educational context. It explores, in turn, its possible extension to the process of institutionalization of other mathematical knowledge in other socio-cultural contexts.
\end{abstract}

Keywords: Social process of institutionalization. Mathematical Knowledge. Theoric and Metodological Model.

\section{Introducción: un modelo desde y dentro de la socioepistemología}

\footnotetext{
* Doctora en Matemática Educativa, Cicata-IPN. Profesora Investigadora del Departamento de Matemática del Consejo de Formación en Educación, ANEP. Uruguay. Enrique Guarnero 3973 CP 11700, Montevideo, Uruguay.E-mail:veromolfino@gmail.com.

Doctora en Matemática Educativa, Cinvestav-IPN. Profesor Investigador del Colegio Mexicano de Matemática Educativa. México. E-mail:buendiag@hotmail.com.
} 
Una de las tareas del matemático educativo es estudiar los fenómenos que se producen en el seno del sistema didáctico cuando los saberes matemáticos constituidos socialmente en ámbitos no escolares se introducen en el sistema de enseñanza. Partimos del supuesto de que la estructura y funcionalidad del saber se modifican continuamente en el transcurso de tal introducción, lo que se detecta al comparar el llamado saber sabio, acuñado por matemáticos en ámbitos científicos, con el saber escolar observable a través del discurso matemático escolar. Más que poner el énfasis en los conocimientos matemáticos presentes en el proceso a través del cuallosobjetos preexistentes se escolarizan, en la investigación que exponemos nos interesa estudiarlos fenómenos de su producción, adquisición y difusión bajo una perspectiva en la que las prácticas sociales de cada uno de los actores involucrados cumplen un rol normativo en la explicación del proceso de trasposición dedichos conocimientos.

Distinguiendo entre la actividad humana, propiamente dicha, y la praxis, que es lo que permite al humano reflexionar sobre dicha actividad, consideramos ala práctica social como un constructo que permite explicar por qué los humanos, individual o grupalmente, actúan de la forma en que lo hacen. En ese sentido es que decimos que la práctica social tiene un carácter normativo (COVIÁN, 2005; CANTORAL et al., 2006). Las prácticas sociales se constituyen, así, en el centro de la explicación de corte socioepistemológico de la trasposición del conocimiento matemático. Existen estudios que explican en particular la transculturación de conocimientos: la forma en que determinados grupos de poder manipulan el conocimiento influyendo sobre su construcción por parte de otras comunidades y permitiendo que el saber viva o no en instituciones educativas externas a dicha manipulación (CAMACHO, 2006). En ese artículo se ejemplifica a través del análisis de libros de texto.

A partir del reconocimiento del hombre haciendo matemáticas en un escenario cultural e históricamente situado, buscamos analizar por qué enseñamos, hoy, a un determinado conocimiento de la manera como lo hacemos. Esa es la pregunta central que ha motivado la creación del modelo teórico-metodológico que se presenta en este artículo.

En particular, proponemos una explicación de por qué se enseña el concepto de límite de la forma en que se hace en el contexto educativo uruguayo a través del estudio de un proceso que denominamos proceso de institucionalización (PI), que permite que se reconozca que lo que el docente enseña en el aula alrededor de ese saber matemático es producto de tal proceso. Si bien la palabra proceso puede sugerir la idea de observar algo en el transcurso del tiempo, el modelo que presentamos propone un corte transversal del sistema en su conjunto, 
que no se caracterice necesariamente por etapas cronológicas, sino que permita una explicación transversal a través de las prácticas de los actores de cada ámbito.

El modelo que proponemos permite describir la naturaleza social del PI que sufre un conocimiento -tomando como ejemplo el concepto de límite-: qué actores intervienen y de qué manera, qué es lo que los hace actuar de la manera en que lo hacen y conduce a determinadas consecuencias en las decisiones sobre qué y cómo se enseña actualmente.

\section{Institución y procesos de institucionalización}

Al reflexionar acerca de por qué se enseña un determinado concepto de la forma en que se hace en el ámbito escolar, es posible reconocer que su construcción no depende exclusivamente de uno o varios individuos, sino que se da a través de las instituciones en las que se desarrolla.

Según Berger y Luckmann (2001, p.76), las instituciones son entidades que posibilitan la conservación de saberes en las sociedades a través del tiempo, como formas organizadas que establecen roles a los participantes, en los cuales existen prescripciones (restricciones) y licencias. Son formas de actuar que norman la conducta de los individuos de una sociedad y se vuelven externas al individuo por los procesos institucionales. Son producto de un proceso de construcción al seno de una historia compartida a través del tiempo y ejercen un control social sobre el comportamiento humano, al establecer pautas definidas que lo canalizan en una dirección determinada en oposición a muchas otras que podrían darse teóricamente. De ellas se desprenden mecanismos de sanción establecidos para el sostén de la institución."La institucionalización aparece cada vez que se da una tipificación recíproca de acciones habitualizadas por tipos de actores".

La investigación en la que se circunscribió la construcción del modelo que presentamos trata con la institución Escuela y la institucionalización de un conocimiento matemático -el límite-en ese ámbito escolar. Molfino (2010) propone un proceso de institucionalización de naturaleza social, ubicandoa las prácticas sociales al centro de la explicación epistemológica de dicha institucionalización para responderpor qué se enseña, hoy, un determinado conocimiento matemático de la manera en que se hace.

Se retoma el modelo epistemológico de prácticas propuesto por Montiel (2011) el cual articula prácticas sociales, prácticas de referencia y actividades para explicar la construcción del conocimiento matemático (figura 1). Una actividad es explícita y se observa en los 
individuos y grupos humanos; el conjunto articulado de actividades intencionales que siguen un propósito específico se denomina práctica de referencia; finalmente, la práctica social se asume como la práctica normativa.En su conjunto dan una explicación acerca de por qué el humano hace lo que hace en escenarios y situaciones específicas.

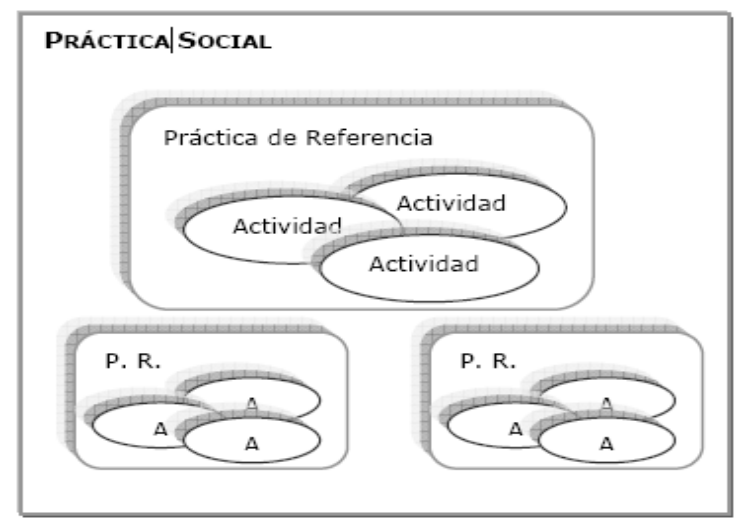

Figura 1 - Un modelo epistemológico de prácticas

Concibiendo de esta manera a las prácticas, Covián (2005) considera a los procesos de institucionalización como aquéllos a través de los cuales las prácticas socialesadquieren la función normativa. En Tuyub y Cantoral (2012) se los utiliza como constructo que evidencia dicha función normativa de la práctica social, analizando qué es lo que favorece las modificaciones y permanencias de determinadas prácticas, inferidas por la continuidad de las mismas, que vuelven institucional un determinado conocimiento.García-Torres y Cantoral (2007) profundizan en los PI, atribuyéndoles la capacidad de explicar la construcción y difusión de conocimiento matemático por parte de la sociedad.

Desde esta perspectiva fue que se planteó el proceso social de institucionalización del concepto de límite (ver figura 2) en Molfino (2010) loque implicó, por un lado, desentrañar cómo un saber matemático -el límite-se constituye como un saber validado, parte de un cuerpo ordenado de conocimientos social y culturalmente aceptados; para ello se analizaron las prácticas sociales inherentes a la evolución del concepto al seno de la comunidad científica matemática (ámbito científico). Por otro lado, se estudió y describiócómo esas prácticas que norman el actuar de los actores del ámbito científico son traspuestas según lo que viven los actores del sistema educativo (ámbito escolar), y cómo,a su vez, norman sus prácticas de referencia, específicas del paradigma en el que actúan. Las actividades concretas observables de los grupos o individuos involucrados son las que permiten describir tales prácticas de referencia a través de las cuales se materializa la manera en que las prácticas sociales norman la construcción de conocimiento. 


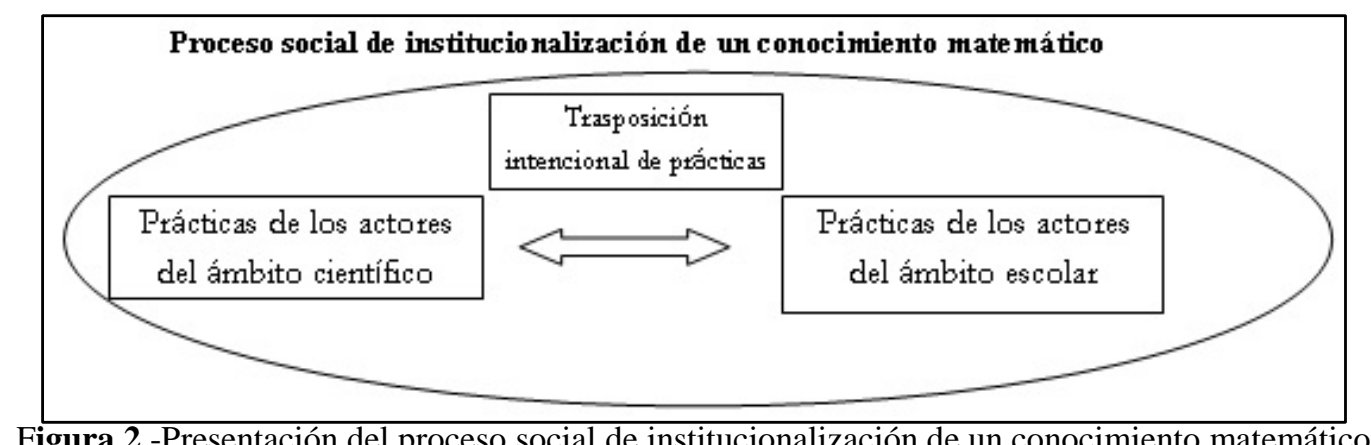

Este análisis implicó la conformación de un modelo teórico-metodológico que considerara la particularidad de cada ámbito. Mientras que el ámbito científico pueden identificarse etapas de una construcción social del conocimiento matemático a la luz de los grupos que se vieron involucrados en tal construcción, en el ámbito escolar interesa más bien identificar momentos de los diferentes grupos que conviven simultáneamente y conforman la institucionalización del concepto en la institución escuela.

\section{La conformación del modelo teórico-metodológico}

Hemos considerado a la socioepistemología como aproximación teórica ya que permite explicar la constitución del conocimiento matemático a través de las prácticas sociales que norman su construcción. Su tarea fundamental es estudiar la construcción de conocimiento situado, aquel que atiende a las circunstancias y a los escenarios socioculturales particulares, caracterizándolo como el fruto de las interacciones entre epistemología y factores sociales (CANTORAL, 2001; BUENDÍA; CORDERO, 2005, CANTORAL; FARFÁN, 2003). De ahí que integre sistémicamente dimensiones de análisis de corte cognitivo, epistemológico, didáctico y social.

El modelo socioepistemológico de prácticas que proponemos permite describir el proceso social de institucionalización a través de prácticas que norman el quehacer de los actores tanto en el ámbito científico como en el escolar. Metodológicamente, lo realizado en cada ámbito requirió de herramientas propias; de manera general, la figura 3 muestra el panorama del modelo propuesto para analizar el proceso social de institucionalización del límite, identificando la articulación entre dos ámbitos. 


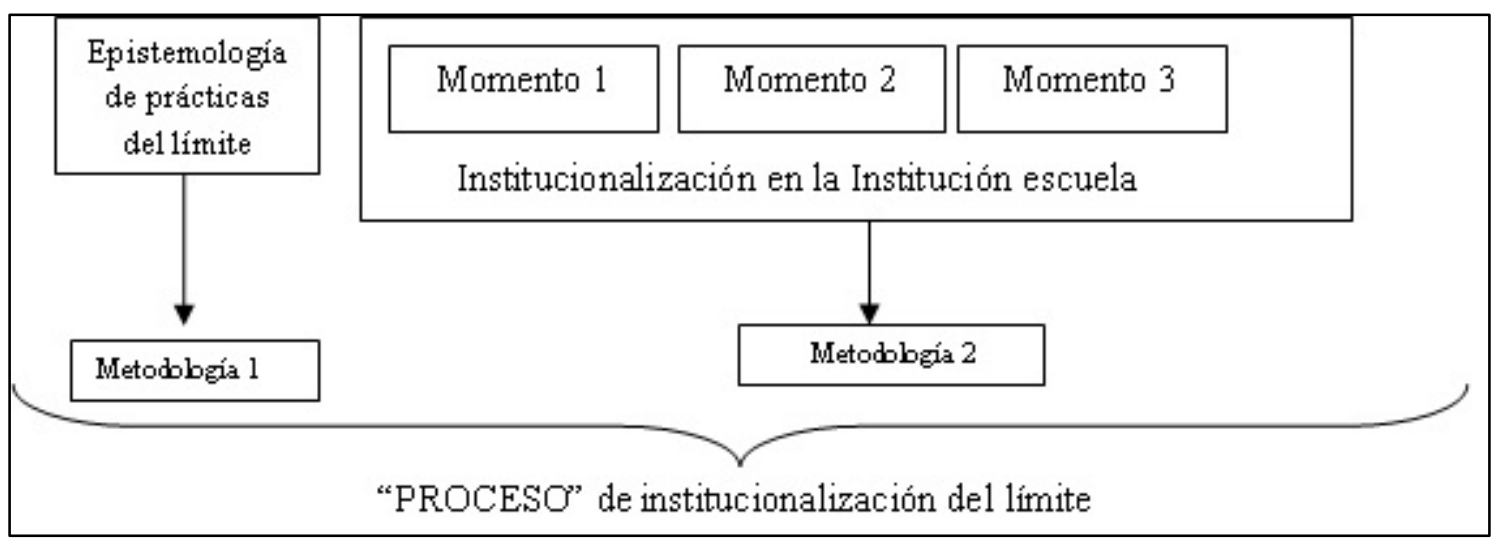

Figura 3- El proceso social de institucionalización

\section{1 Ámbito científico: epistemología de prácticas}

En el ámbito científico, se realizó una búsqueda de carácter epistemológico-histórico sobre el concepto límite de una función real. Lejos de caracterizarse por una sucesión de hechos históricos alrededor de la evolución del concepto, la búsqueda es sobre aquellas circunstancias que rodean el quehacer del hombre relativo al desarrollo de dicho concepto. La historia se vuelve una fuente de significados (CORDERO, 2001; BUENDÍA; MONTIEL, 2011; BUENDÍA; CORDERO, 2005) y el interés no es la producción matemática final lograda, sino el análisis de la actividad humana al hacer y usar matemática en contextos sociales específicos.

En Molfino y Buendía (2010) se estudió, entonces, la evolución histórica del concepto de límite, pero tratando de detectar prácticas que determinaran los diferentes momentos dentro de la evolución del concepto. Así mismo, se analizó qué necesidades científicas condujeron a la configuración del concepto en cada una de ellas. Es decir, se buscaba analizar por qué el concepto se constituyó de determinada manera -y no de otra-en determinado ámbito. Es por este doble rol -generador de conocimientos y normativa del actuar humano-que proponemos considerarlas como prácticas sociales.

Este análisis permitió reconocer que el concepto de límite surge como una herramienta que permite formalizar, generalizar y difundir resultados, prácticas que promovieron el desarrollo de actividades matemáticas en cada etapa como el cálculo de áreas y volúmenes, la 
refutación o demostración rigurosa a través del método de exhausión, el cálculo de máximos y mínimos en problemas que respondían a necesidades de cada etapa, el trabajo con métodos infinitesimales, la formalización del concepto de función, la confección de libros de texto y dictado de cursos, la extensión y generalización del concepto a nuevos contextos matemáticos, la conjeturación y demostración de teoremas, entre otros. Sin que necesariamente sean explícitas en cuanto a objetivos a cumplir por un matemático determinado, sí cumplieron un papel normativo en el quehacer de los diferentes grupos sociales que participaron en la actual configuración del concepto.

\section{PS: FORMALIZACIÓN}

PR: Mate matizar el cálculo en ambiente geométrico-estático.

Actividades:

- Calcular áreas y volúmenes.

- Refutar o de mostrar rigurosamente por exhausión.

PS: FORMALIZACIÓNGENERALIZACIÓN-DIFUSIÓN

$\underline{\text { PR: }}$ Aritmetizar el análisis en ambiente algebraico-analítico.

Actividades:

- Resolver nuevos problemas.

- Reconstruir el concepto de función.

- Confección de libros de texto.

- Dictado de cursos.

\section{PS: PREDICCIÓN}

PR: Mate matizar el movimiento en ambiente geométrico-dinámico. Actividades:

- Buscar resultados para problemas concretos.

- Trabajar con métodos infinitesimales.

- Priorizar la intuición.

\section{PS: GENERALIZACION}

PR: Generalizar el análisis a contexto topológico.

Actividades:

- Considerar nuevas conjeturas.

- Extender a nuevos contextos

- Enunciar nuevas definiciones.

- Demostrar nuevos teoremas.

Figura 4 - Un modelo epistemológico de prácticas para el límite

Reconocemos, así, que el concepto de límite no está conformado por conceptos y estructuraciones conceptuales aisladas, sino que es fruto de una articulación de actividades humanas gestada al seno de dichas prácticas. Resumimos el modelo epistemológico de prácticas relativo al concepto de límite funcional en la figura 4.

\section{2 Ámbito escolar: análisis crítico del discurso}

Con el fin de analizar cómo las prácticas sociales detectadas norman el quehacer de los actores en el ámbito escolar, estudiamos el discurso matemático escolar reflejado a través de algunas de sus actividades concretas: programas, libros de texto, entrevistas y cuestionarios a docentes. Según explican Cantoral et al. (2006), el discurso matemático escolar (dme) está conformado por los discursos relacionados con la difusión de un saber matemático. La 
estructura de dichos discursos no se reduce a la organización de los contenidos temáticos o programas, ni a su función declarativa en el aula a través del discurso del profesor, sino que implica el establecimiento de bases de comunicación para la formación de consensos y la construcción de significados compartidos.

Es desde esta perspectiva, y entendiendo al discurso como modelador de las acciones, que proponemos analizar, a partir del dme, las prácticas que se generan en el tránsito entre los ámbitos científico y escolar y, posteriormente, al seno del escolar mismo. Esto permite explicitar el papel de las prácticas escolares, entendidas como las prácticas de referencia en el ámbito escolar.

Para ello, interesa identificar el rol que el dme cumple en la construcción de conocimiento, de tal manera que se reconozca que lo que el docente enseña en el aula alrededor de un determinado saber matemático es producto de ese proceso social de institucionalización. Se propone entender al dme como una acción social y analizarlo como un fenómeno social y cultural, con el fin de comprender las relaciones entre él y la sociedad. Optamos, entonces, por el Análisis Crítico del Discurso (ACD), herramienta metodológica utilizada en estudios donde el marco teórico implica entender al discurso como acción social (VAN DIJK, 2001).

El ACD es un tipo particular de investigación analítica sobre el discurso que estudia el modo en que el abuso del poder social, el dominio y las desigualdades son practicados, reproducidos y combatidos por los textos y el habla en el contexto social y político. Busca establecer nexos entre las propiedades de un texto y las estructuras y procesos sociales y culturales (FAIRCLOUGH; WODAK, 2001). Sus ocho principios básicos ${ }^{1}$ fueron descritos en Molfino y Buendía (2011a), en donde se ejemplifican para el caso particular del análisis del proceso social de institucionalización del concepto de límite.

En el ámbito escolar, el modelo epistemológico de prácticas permite distinguir momentos, ya no en el sentido cronológico de las etapas, sino como diferentes fases que se pueden distinguir en el proceso social de institucionalización cuando reinterpretamos las prácticas sociales que norman las prácticas de referencia de los actores del sistema educativo y la sociedad en su conjunto. Esos momentos se describen en función de las prácticas de los actores que participan con más protagonismo en cada uno y de los roles que dichos actores

\footnotetext{
${ }^{1}$ 1. El ACD se ocupa de los problemas sociales. 2. Las relaciones de poder son consideradas como elementos discursivos. 3. El discurso constituye a la sociedad y a la cultura. 4. El discurso realiza una labor ideológica. 5. El discurso es histórico. 6. El vínculo entre el texto y la sociedad es mediado. 7. El análisis del discurso es interpretativo y explicativo. 8. El discurso es una forma de acción social.
} 
desempeñan. Este análisis es, por consiguiente, situacional para la institución escolar analizada.

Para analizar la inclusión del concepto de límite en el ámbito escolar en el contexto educativo uruguayo, estudiamos la evolución de los programas del curso de Matemática de último año de Educación Secundaria en Uruguay, específicamente de las opciones Medicina e Ingeniería. También se analizaron los programas vigentes y los anteriores de Formación de Profesores de Matemática, centrando en todos los casos el análisis en el concepto de límite, con el fin de conocer cuál era la expectativa desde las autoridades educativas sobre la enseñanza del tema, y entender más la situación de los profesores al respecto. Este análisis también permitió observar qué libros de texto son recomendados por las autoridades educativas en matemática (en caso de que se recomendara alguno), que son quienes confeccionan los programas.

Por otro lado, se analizaron libros de texto, tanto actuales y disponibles en plaza en Uruguay, para su uso en los cursos de secundaria, como los libros de texto de antaño, utilizados como referencia en los cursos de Secundaria y Formación Docente desde la segunda mitad del siglo XX. Se analizaron, también, los apuntes que un profesor del Instituto de Profesores de Montevideo confeccionara para sus estudiantes, el cual es de suma importancia por su influencia sobre las concepciones y creencias de futuros docentes sobre lo que es el Cálculo y su enseñanza, así como sobre sus prácticas educativas futuras.

Además, se implementaron cuestionarios y entrevistas a docentes. En primer lugar se confeccionó y aplicó un cuestionario a docentes del curso de Matemática del último año de Educación Secundaria. Algunas de sus preguntas buscaban que el docente explicitara su tratamiento didáctico del límite: ¿Qué definición presenta? ¿Es la única? ¿Trabaja con alguna equivalente?; ¿Presenta algún ejemplo? ¿En qué contextos: tabular, gráfico, situación extramatemática, analítico-algebraico?; ¿Propone alguna actividad en torno al concepto de límite previo a introducir la definición?; ¿Realiza alguna actividad para promover el uso de cuantificadores o símbolos lógicos en general?; ¿Qué libro de texto recomienda? ¿Lo utiliza en clase para trabajar este tema o para proponer ejercicios referidos al mismo?Otras preguntas brindaban la oportunidad al docente de reflexionar sobre las dificultades que ese abordaje le acarrea a sus estudiantes, tanto en el transcurso de la clase como en evaluaciones estructuradas: ¿Qué dificultades detecta en los estudiantes al trabajar con esta definición?; ¿Qué obstáculos debe afrontar un estudiante que no comprende la definición de límite? ¿En qué "fallaría” o qué otro concepto le imposibilitaría construir? ¿Cuál es el tipo de pregunta 
que realiza habitualmente en evaluaciones orales o escritas que el estudiante no responde o lo hace erróneamente por no manejar la definición de límite? Dado que el cuestionario se realizó a través de preguntas explícitas y directas, las respuestas de los docentes permitieron hacernos una idea de sus concepciones en torno a la enseñanza del límite. Estas concepciones dan cuenta, por un lado, de una manera particular de entender el por qué debe ser enseñado el concepto de límite en Educación Secundaria y cómo, en un contexto y época determinados. El docente las va conformando a lo largo de su formación, en la discusión con colegas y autoridades y en reuniones de coordinación. El tipo de preguntas que pretendía explicitar dichas concepciones eran, por ejemplo, ¿cree que es importante que esté este contenido en el programa de $6^{\circ 2}$ ? ¿Por qué? o ¿Con qué problemática cree que se encontraría en el transcurso del curso si en lugar de la definición utilizara un acercamiento intuitivo al concepto? Estos cuestionarios fueron enviados por correo electrónico a varios profesores, contactados previamente en forma personal para explicar el propósito del mismo y su tratamiento en la investigación. Esto permitió que se tomaran el tiempo que ellos creyeran necesario para elaborar las respuestas. De ellos, diez contestaron.

Por otra parte, se ha justificado que en Uruguay ha influido fuertemente la oralidad en la perpetuación del discurso escolar, predominando en ocasiones sobre aspectos escritos del mismo (MOLFINO, 2010). Ello se debe, en parte, al difícil acceso a los libros de texto. Es por eso que se realizó una entrevista a un profesor con cincuenta años de experiencia en Secundaria, Formación Docente y Universidad (en adelante, Docente 1), la cual fue de suma importancia para extraer evidencias, ya que el docente se detiene en experiencias acerca de cómo influían los libros de texto y los apuntes de clase sobre las prácticas de aula. Por su vasta experiencia, se consideró que podía representar un testimonio significativo para la descripción de los procesos sociales de institucionalización.

Si bien se dio a optar entre modalidades presenciales de contacto, el profesor optó por responder vía correo electrónico. En principio se le preguntó sobre cuáles han sido los textos que a lo largo de su trayectoria como docente habían sido utilizados como referencia para el planeamiento de cursos y en qué años, aproximadamente. Entre otras cuestiones, el docente afirmó que en sus estudios secundarios, "me apoyé fundamentalmente en los apuntes de clase

\footnotetext{
${ }^{2}$ En el sistema educativo uruguayo, lo que antiguamente se denominaba $6^{\circ}$ año de Educación Secundaria corresponde a lo que actualmente se denomina 3er año de Bachillerato, que es el último de Educación Secundaria. Los estudiantes tienen generalmente entre 17 y 18 años. Si bien oficialmente hubo un cambio en el nombre del nivel, por cuestiones de tradición los profesores, estudiantes y sociedad en general siguen denominándolo $6^{\circ}$ año.
} 
y en juegos de apuntes a mimeógrafo de algunos de los principales profesores del IAVA ${ }^{3}$. Es muy difícil que haya recurrido a los libros que te mencioné (ni nadie lo hacía ya que eran bastante diferentes al desarrollo de los cursos, que eran bastante fuertes)"(DOCENTE 1).

Eso motivó un nuevo cuestionamiento: ¿Por qué es que los profesores del momento insistían tanto con cursos tan fuertes que ni siquiera los libros de texto que había en el momento se adecuaban a esos cursos? y ¿en qué se basaban esos apuntes, es decir qué los inspiraba: alguna influencia de la manera de enseñar en algún otro país, por ejemplo, o qué? Una de las causas brindadas por el docente para la existencia de tales apuntes es la poca disponibilidad de los libros de texto que se tenía en ese entonces. También explica que algunos apuntes incluían temas que en tales libros de texto o bien no eran abordados o eran abordados con un enfoque diferente al que pretendía el docente.

Por último, el análisis de los programas y su evolución condujo a cuestionarnos acerca de la manera en que los profesores aceptan las modificaciones, y lo traducen en nuevos abordajes para el tratamiento del concepto de límite en el aula. Para indagar sobre la forma en que son aceptados dichos cambios por parte de los docentes, se llevaron a cabo dos entrevistas a docentes de secundaria(en adelante, Docentes 2 y 3 , respectivamente) con vasta experiencia en el dictado del curso de Análisis para sexto año de Educación Secundaria que oficiaron de testimonios de algunas de las concepciones de enseñanza de la matemática presente en el sistema educativo uruguayo. Se buscaba analizar cómo los docentes sintieron este cambio, para buscar evidencias sobre los diferentes momentos en el proceso social de institucionalización del concepto de límite.

En esas entrevistas se preguntaba, en primer lugar, ¿Hace cuánto tiempo que se recibió? ¿Hace cuánto tiempo que se desempeña como profesor de Matemática? Esto con el fin de conocer si su trayectoria incluía suficiente experiencia previo al cambio programático, dado en 2008 y evaluar cuánto influía en su discurso el peso de la tradición anterior. Más adelante, se preguntaba sobre su opinión sobre dichos cambios programáticos: ¿cuáles cambios le parecen a Ud. más significativos con respecto al programa de Análisis en la opción Ingeniería? ¿Y cómo le parece que eso afecta en la manera en que se propone el tema límite de funciones? ¿Hubo un cambio en eso?

\footnotetext{
3 “Instituto Alfredo Vásquez Acevedo" (IAVA): Inaugurado en 1911 como Sección de Enseñanza Secundaria y Preparatoria de la Universidad, fue el único instituto de educación media superior (Preuniversitaria) hasta la década de 1940, lo que lo constituía en la institución que nucleaba todos los estudios preuniversitarios. En 1935, se independiza la Educación Secundaria de la Universidad, pero el IAVA siguió cumpliendo sus funciones como institución de Educación Secundaria, hasta el día de hoy (http://iava.edu.uy/historia.htm).
} 
A continuación se realizaron una serie de preguntas para conocer si estos cambios programáticos influyeron en el discurso del profesor, y de qué manera: la manera en que planifica y lleva a cabo el curso, ¿se modificó respecto a la manera en que lo hacía dos años atrás? Se preguntó específicamente sobre el tratamiento del concepto de límite.

Y, por último, se buscaba identificar cuáles son los usos que el docente da al concepto de límite en sus prácticas escolares: ¿Por qué cree importante el tratamiento del tema límite de funciones dentro del curso tal cual lo plantea? Con base en el cuestionario y entrevistas a docentes, se buscó reunir elementos sobre cuál es el papel que los profesores le asignan al límite en la estructuración de su curso; esto es, si consideran que la definición es inamovible, sustituible, imprescindible para la formación del estudiante, innecesaria o cualquier otra alternativa. También se pretendió dar cuenta de en qué medida el límite representa en esas prácticas docentes un rol de estructurador del Cálculo: qué tan importante es su tratamiento para la construcción y articulación de otros conceptos, como el de continuidad, derivada o integral.

A su vez, se deseaba indagar acerca de cómo influyen las exigencias de los programas en las decisiones docentes y cuál es el papel que los libros de texto desempeñan en el tratamiento escolar del concepto de límite. Explicitar estos elementos daría cuenta de las prácticas de los docentes como agentes de la noósfera acerca de la manera en que es abordado, hoy, el concepto en clase y de las posibles alternativas para hacerlo.

\section{El proceso social de institucionalización del límite}

Al entender el discurso matemático escolar alrededor del límite de funciones como acción social podemos entender cómo aquellas prácticas sociales formalización, generalización y difusión-norman las prácticas de referencia de los actores del ámbito escolar. Ello dio lugar a la identificación de tres momentos en este proceso social de institucionalización: diferentes fases que se pueden distinguir cuando queremos reinterpretar las prácticas sociales en la institución escuela.

Los mismos pueden describirse en función de los actores de la noósfera, que participan con más protagonismo en cada uno, y de los roles que dichos actores desempeñan:

a) el tránsito del concepto límite funcional desde la comunidad científica hacia la escuela;b) las interacciones entre docentes y entre docentes y autoridades educativas; y c) el desarrollo del concepto de límite en el aula. 
Estos momentos transcurren simultáneamente y no uno a continuación del otro. Cualquiera de ellos es dinámico en el tiempo e influye continuamente sobre los demás.

Para la distinción de tales momentos partimos del supuesto de Van Dijk (2001) referido a la multiplicidad de acciones que se realizan al hablar o escribir -defender una postura, responder preguntas o representar a tal grupo social, por ejemplo. Así, por ejemplo, entendemos que los profesores entrevistados o que completaron el cuestionario son parte de un grupo social, el de los profesores de matemática de Uruguay, que, a su vez, defienden una postura determinada, que puede ser diferente a la de otros integrantes del grupo. Lo mismo ocurre con los actores involucrados en la confección de los programas o en la escritura de los libros de texto, con los matemáticos de la comunidad científica, así como con el resto de los actores de la noósfera involucrados en el proceso social de institucionalización.

\subsection{Momento a: tránsito del concepto límite funcional desde la comunidad científica hacia la escuela}

En este momento, encontramos dos grandes grupos de poder que influyen sobre el quehacer docente: las autoridades de educación matemática y la comunidad de matemáticos, propiamente dicha. Con respecto a la primera, nos centramos en la Inspección de Matemática, organismo encargado entre otras cosas de la confección de los programas y recomendación de libros de texto, es decir, la agenda de lo que los profesores deberían hacer y decir en el aula. Respecto de los programas, observamos que en el confeccionado para la opción Ingeniería de 1976 (anterior al vigente) es una lista de contenidos, en este orden: Número Real, Sucesiones, Funciones, Funciones continuas, Funciones derivables, Funciones continuas en intervalos, Funciones derivables en intervalos, Funciones inversas, Estudio de funciones, Series numéricas y Aproximación de Funciones por polinomios. No se explicitan objetivos, cargas horarias ni sugerencias metodológicas. Esto denota un abordaje tradicional del Cálculo, en el que el límite se erige como contenido estructurador del resto de los conceptos. Este abordaje, según constatamos con la entrevista al docente 1, se propuso como una continuación de prácticas que ya se llevaban a cabo desde mediados de siglo XX en el Uruguay: énfasis en los aspectos formales del curso, influenciados fuertemente por la etapa de aritmetización del análisis en la evolución del concepto de límite. El programa vigente para la misma orientación desde 2008 pretende problematizar tal posición, proponiendo algunos cambios en la modalidad de trabajo (exigiendo que se le dé mayor participación al estudiante y 
oportunidades de conjeturar y demostrar o refutar por sus propios medios) y explicitando objetivos generales y específicos del curso, como una mayor integración de los aspectos prácticos y teóricos a través del planteo de situaciones problemáticas, exigiendo una menor formalización en la justificación de afirmaciones y una mejor articulación entre aspectos gráficos y numéricos alrededor de los conceptos tratados en el curso. Sin embargo, no apreciamos una problematización de los contenidos a abordar propiamente dichos: se mantiene al concepto de límite como estructurador de los demás conceptos: continuidad, derivadas e integrales.

En este aspecto tiene una gran influencia el otro grupo mencionado, la comunidad matemática uruguaya e internacional, tanto en forma explícita a través de consultas puntuales para el diseño de programas y lineamientos, como a través de la confección de libros de texto. Estas relaciones de poder también se explicitan en la confección de libros de texto como acto discursivo: en el estudio fue constatado que los actuales libros de texto de Análisis o Cálculo están basados en los que nosotros denominamos de antaño en cuanto a su estructura formal, y éstos, a su vez, son libros extranjeros, que imponen determinadas prácticas escolares que no fueron pensadas para la situación específica de Uruguay ni para la actualidad (MOLFINO; BUENDÍA, 2011b).

Los autores de libros de texto -mayormente matemáticos y profesores de matemáticase constituyen, pues, en otro grupo de poder que influye sobre el tránsito del concepto de límite de la comunidad científica a la escolar. Sugieren, a través de esos elementos discursivos, una determinada ideología (influenciada a su vez, fuertemente, por la comunidad científico-matemática) acerca de cómo se debe enseñar la matemática, cuáles son los contenidos a tratar y cuál es la estructuración que se le debe dar a los mismos.En los libros considerados de antaño (REY PASTOR, 1917; REY PASTOR; PI CALLEJA; TREJO, 1952), se priorizan los métodos deductivos por sobre los inductivos y la utilización del lenguaje simbólico y formal por sobre el coloquial o gráfico, en forma intencional y explicitada en el segundo libro mencionado. El autor pretende mostrar una exposición sistemática, lógicamente encadenada. Se evitan, intencionalmente, detalles relativos a la historia de la ciencia o disquisiciones metafísicas, considerados superfluos o secundarios. En los libros actuales, si bien se explicita una intención por utilizar un lenguaje simple que evite el uso de cuantificadores y por priorizar el entendimiento al excesivo formalismo y las introducciones intuitivas, se mantiene la estructura formal, en donde los conceptos de límite y número real son definidos formalmente para sostener la estructura del análisis de funciones 
desarrollada posteriormente. A modo de ejemplo de lo paradójico de la intención explicitada, observamos que presentan, como definición intuitiva de límite, la siguiente, que no es más que la definición formal, expresada en forma verbal (GIOVANNINI, 2001, p.58):

Se dice que el límite de $\mathrm{f}(\mathrm{x})$ es $\mathrm{b}$ para $\mathrm{x}$ tendiendo al valor a y lo notaremos $\lim _{x \rightarrow a} f(x)$ si para todo entorno de centro $b$, existe un entorno reducido de centro a, inluido en su dominio, tal que: si $\mathrm{x}$ pertenece a ese entorno reducido los correspondientes valores funcionales pertenecen al entorno de centro $b$.

Por último, la primera de las entrevistas descriptas brindó evidencias sobre la manera en que el concepto de límite llegó a nuestras aulas de la forma en que es tratado hoy en día, desde la visión del profesor entrevistado. Pudo constatarse que, si bien algunos libros de texto fueron influyentes para la conformación de los primeros cursos (muy apegados a la formalidad requerida por la comunidad matemática), uno de los aspectos que más influyó fue la difusión de apuntes de clase de prestigiosos docentes. Esos apuntes constituyeron una fuente validada para la preparación de clases durante muchos años, contribuyendo, de esa manera, a que el saber desarrollado en el ámbito académico se difundiera en el escolar:

La principal herramienta de estudio para el teórico eran los apuntes individuales que sacábamos cada uno de nosotros [haciendo referencia a sus estudios de Secundaria]. Los "Apuntes de ciertos docentes" se recomendaban como complemento de lo dado en clase(...) Por ejemplo existían unos "Apuntes del Prof. Forteza" que fueron confeccionados por un grupo de estudiantes que sacó apuntes del curso de Forteza, luego los pasó en limpio y Forteza le hizo una revisación final, y así se imprimieron(...)Con esto te estoy diciendo que los mismos no fueron redactados con una intención especialmente didáctica sino como resumen de los temas dictados (DOCENTE 1).

Llama la atención cómo el docente denomina teóricos a los estudiantes de nivel secundario, lo que deja entrever un sentimiento de pertenencia a la comunidad matemática. Esto podría ser influenciado por la forma en que se dictaban los cursos en ese momento. Debemos considerar, al respecto, que la comunidad de estudiantes y profesores era muy reducida, basta pensar que hasta los años 60existía un único instituto público en Uruguay que ofrecía estudios preparatorios (preuniversitarios, actualmente en la órbita de la Educación Secundaria, denominados Bachilleratos). Esto favorecía una mayor cohesión entre las prácticas de los diferentes docentes, apoyadas en los mencionados apuntes, sin finalidad didáctica explícita. También se desprende del siguiente fragmento:

En primer lugar los cursos de matemática de Facultad de Ingeniería eran "fuertes desde fines de la década de los 30', y allí no se daba límite de sucesiones ni funciones reales, sino funciones de varias variables, funciones de variable compleja $\mathrm{y}$ ecuaciones diferenciales; el cálculo diferencial de una variable se dejaba para 
Preparatorios. Varios de los docentes de Preparatorios eran docentes de la Facultad o habían sido estudiantes de Ingeniería (DOCENTE 1).

Otro aspecto de influencia de la comunidad matemática sobre la escolar se evidencia a través de las prácticas ejercidas en la Universidad (en particular en la Facultad de Ingeniería). En la entrevista realizada a docentes para analizar la influencia de cambios de programas, uno de ellos expresa dicha influencia, en relación al concepto de límite:

Me parece que es un tema de capital importancia y además en la Universidad nos piden, entonces en la medida en que nosotros no hagamos una conjunción con la Universidad, que siempre nos hemos llevado a las patadas, la verdad... de pronto pueden decir "no den tanto de límite, den sólo el concepto, la definición, y nosotros nos encargamos de..." Pero a mi me parece que el tema en sí, es un tema muy rico, y que habilita justamente a muchas definiciones posteriores (DOCENTE2).

Este análisis ha permitido constatar que en la transición del ámbito científico al escolar ha influido fuertemente el paradigma desarrollado en la cuarta etapa del ámbito científico del proceso social de institucionalización. La conformación del concepto de límite en el ámbito escolar se ve fuertemente normada por las prácticas sociales de formalizar y generalizar, conformando una determinada manera de estructurar el cálculo y un determinado rol del límite como eje central de dicha estructuración.

\subsection{Momento b: interacciones entre docentes y entre docentes y autoridades educativas}

En este momento estudiamos la relación de poder que las autoridades educativas entablan, a través de su discurso y de sus prácticas, con los docentes. Los inspectores de Matemática son quienes se encargan de difundir los programas y su implementación a través de reuniones con docentes. En estas reuniones se sugieren determinados lineamientos en cuanto al orden y jerarquía de los temas y sugerencias metodológicas. A su vez, estos mismos inspectores evalúan a los docentes durante el año lectivo, a través de visitas puntuales (se observa una hora de clase y se analiza la libreta del profesor, libreta en la cual el docente debe llevar anotado el desarrollo del curso y las evaluaciones de sus alumnos). El proceso de institucionalización del límite, fundamentándose en la versión oficial del mismo, toma vigencia en tanto poder de los inspectores, debido a que de ellos puede depender la continuidad laboral de un profesor.

También son importantes, a los efectos de entender este momento en el proceso social de institucionalización del concepto de límite, las reuniones de coordinación que se dan a la 
interna de cada instituto de enseñanza media. Allí participan profesores de Matemática del mismo instituto, pero con diferentes grados de experiencia en el dictado de cursos, lo que los posiciona en diferentes roles en cuanto a la toma de decisiones sobre cómo dar determinados temas (en particular el de límite). Si bien este es un aspecto difícil de analizar explícitamente, ya que forma parte de lo que anteriormente denominamos como tradición oral, puede constatarse la influencia que profesores con más experiencia ejercen sobre los que se inician en la profesión, a través de la recomendación de la utilización de determinados repartidos (listados de ejercicios), apuntes, libros de texto o abordajes en general. En este sentido, los docentes más jóvenes pudieran verse obligados a seguir determinada estructura en cuanto a la organización y jerarquización de contenidos amén de poder coordinar, a fin de año, una evaluación común a los diferentes grupos del mismo grado para ese instituto. En este momento de institucionalización de prácticas resulta evidente la influencia de las diferentes concepciones sobre el concepto de límite que conviven en los profesores: en algún sentido se fortalece la tradición oral (tanto por las reuniones descriptas en el párrafo anterior como por la propia formación de profesores, caracterizada por una gran carga de práctica docente en la que se observan clases de profesores considerados idóneos) pero en otro, se gesta la búsqueda por alternativas, que se explicita en el siguiente momento.

\subsection{Momento c: desarrollo del concepto de límite en el aula}

Si bien los docentes participan, de alguna manera, en los otros dos momentos, es en este en el que el colectivo docente se erige como un grupo de poder protagonista en la institucionalización del concepto de límite. Así como los docentes son evaluados por los inspectores, también los estudiantes son evaluados por los docentes, lo que sugiere un interés por parte del estudiante de aprender y lograr reproducir el discurso que el docente le ofrece. Algunos de los docentes que respondieron al cuestionario propuesto en Molfino (2010) manifiestan no evaluar directamente la definición de límite, pero los que lo hacen lo evalúan atendiendo a aspectos formales, del tipo aplicar la definición de límite para demostrar que un límite dado es un número dado o cambio en el orden de los cuantificadores para que los estudiantes detecten la diferencia, o la existencia o no de la imagen de un punto del dominio en el que no existe el límite de la función. Por su parte, en los libros de texto se atienden más bien aspectos algorítmicos del concepto de límite para la evaluación, prácticamente no se evalúa en forma explícita la definición sino la operatoria de límites. 
El discurso de los docentes constituye a la sociedad y a la cultura, a la vez que es constituido por ellas dado que el docente es miembro de un grupo social, y que como tal sus concepciones son influidas por las del grupo y las exigencias externas que tiene ese grupo. Ello se traduce en un conflicto que pudo entreverse en las respuestas de algunos docentes al cuestionario. Por un lado, sostienen que es importante la introducción del concepto de límite porque es central en la estructuración del cálculo para el desarrollo de otros conceptos como el de derivada, transmitiendo una concepción del límite normada por las prácticas de formalización y generalización. En este sentido, los docentes se ven influenciados por lo que las autoridades plantean en los programas, por la presentación del tema en los libros de texto y, muy probablemente, por su formación inicial: no sólo por cómo estaba estructurado el tema en los currículos escolares de su época sino, además, por las prácticas docentes de sus propios profesores. Pero, por otro lado, los docentes que responden al cuestionario reconocen que este es un tema difícil para los estudiantes por el grado de abstracción que requiere, y que por eso sería posible pensar en no realizar un tratamiento formal del mismo en cursos de opciones que no requieran una carga horaria extensa de matemática, como la opción Derecho, por ejemplo. Eso podría estar dando cuenta de la influencia de las exigencias de un mayor índice de aprobación por parte de la sociedad.

Puede apreciarse, también, la labor ideológica del discurso en las entrevistas realizadas a raíz del cambio en los programas. Por un lado, si bien en los programas vigentes se muestran indicios de quitar importancia a la formalización en instancias introductorias del Cálculo (como el curso de último año de Educación Media), entrevistas realizadas a docentes permitieron constatar cierta resistencia a esa propuesta, con respuestas como: "[...] todo lo que tiene que ver con la parte de Análisis de las funciones, la continuidad, las sucesiones, yo le doy la misma formalidad que antes. Así que yo trabajo igual." (DOCENTE 3). El cambio en el enfoque metodológico y conceptual de los programas constituye en sí una labor ideológica, ya que se pretende modificar un producto institucionalizado, aceptado durante años por el grupo de docentes de matemática. Se pretende con ellos asegurar que el grupo de docentes desarrolle prácticas educativas diferentes a las previamente apropiadas, similares entre sí, favoreciendo, de esa manera, su cohesión. Pero características específicas del cuerpo docente uruguayo hacen que sea difícil el cambio de propuesta, incluso cuando es sugerida en el programa oficial (MOLFINO, 2010). Por otro lado, la resistencia que los docentes manifiestan sobre algunos aspectos del programa sugiere una determinada ideología persistente en su discurso, caracterizada por la necesidad de formalizar los conceptos con los que se trabaja en 
el curso, y que contribuye a que los docentes mantengan prácticas educativas características de la metodología de trabajo de los programas anteriores, según ellos mismos manifiestan.

En suma, a través del análisis del proceso social de institucionalización en diferentes momentos hemos detallado el papel de las prácticas de los actores de la noósfera involucrados en cada uno de ellos y de las relaciones de poder que los vinculan. Esto nos ha permitido abordar la pregunta acerca de por qué se enseña el concepto de límite, en el ámbito educativo uruguayo, de la manera en que se hace. Hemos detectado cómo las prácticas de formalizar, difundir y generalizar norman el quehacer de autoridades, escritores de programas y libros de texto y docentes a través de diversos actos discursivos como los textos, programas, apuntes, reuniones entre diferentes grupos de actores, observación y dictado de clases, entre otros.

\section{Posibles aplicaciones del modelo en otros contextos}

Consideramos que el modelo teórico-metodológico propuesto tiene varios aspectos que pueden ser útiles para analizar el proceso social de institucionalización de otros conceptos matemáticos, en diferentes escenarios socioculturales.

La importancia y viabilidad de elaborar epistemologías de prácticas está vastamente evidenciada a través de una cantidad considerable de investigaciones realizadas desde la perspectiva socioepistemológica. A tales efectos, basta consultar las Actas Latinoamericanas de Matemática Educativa más recientes (FLORES, 2011; LESTÓN, 2011) para conocer cómo se lleva a la práctica tal constructo, así como los trabajos más recientes que instruyen sobre sus fundamentos teóricos (BUENDÍA; MONTIEL, 2011). Esa sería la parte del modelo que permitiría explicitar las prácticas sociales que norman el actuar de quienes conforman la comunidad científica.

En el caso del concepto de límite, vimos cómo algunas prácticas sociales influyeron, específicamente, en el proceso de su constitución en el ámbito científico: para otros conceptos pueden ser otras tales prácticas. Pero, lo interesante del modelo que aquí proponemos, es que permite explicitar cómo esas prácticas sociales norman también el quehacer de los actores del ámbito escolar. 
Ello se llevó a cabo utilizando como herramienta teórica la consideración del discurso como una acción social (VAN DIJK, 2001) y su correspondiente herramienta metodológica, el ACD. Todos los actores que consideramos para el proceso social de institucionalización del concepto de límite, son comunes a los procesos en los que otros conceptos se han institucionalizado en el contexto educativo uruguayo. Incluso podría pensarse que la identificación de los momentos para el ámbito escolar puede ser muy similar, para otros conceptos, en el mismo escenario sociocultural.

Es probable que en otros escenarios, fuera del ámbito del sistema educativo uruguayo de Enseñanza Media, sean otros los momentos relevantes a identificar, y otros los actores a considerar, pero su descripción podría hacerse a través del ACD como herramienta metodológica: entendiendo a cada acto discursivo, escrito u oral, como una acción social, emitida por personas que a su vez pertenecen a determinados grupos, y que pueden estar actuando como representantes de sus intereses y supuestos aceptados como válidos a la interna de ese grupo.

En el marco de un curso para profesores egresados en Uruguay, se ha realizado la experiencia de aplicar la herramienta del ACD al discurso escolar en torno al uso de las gráficas, en particular al quehacer docente normado por la graficación como práctica social, en el sentido en que lo plantean Cordero y Flores(2007). Por razones de extensión y exigencias del curso, los trabajos realizados fueron, en general, analizando en forma parcial alguno de los elementos discursivos: analizaron el discurso de un docente a través de la observación de una sola clase y de la consideración de qué libros de texto utiliza. Pero lo recabado hasta el momento es muy prometedor: en particular, uno de los trabajos realizó un interesante paralelismo entre lo que los programas oficiales sugerían para el dictado del tema sobre el cual versó la clase y la manera de actuar del docente en la misma. Estos incipientes trabajos sugieren que el modelo puede ser extendido al análisis del proceso social de institucionalización de otros conceptos en el mismo contexto sociocultural, está aún por experimentarse si también puede ser extendido a otros contextos.

\section{Referencias}

BERGER, P.; LUCKMANN, T. La construcción social de la realidad. Buenos Aires: Amorrortu Editores, 2001. 240p.

BUENDÍA, G.; CORDERO, F. Prediction and the periodic aspect as generators of knowledge in a social practice framework. A socioepistemological study. Educational Studies in Mathematics, Kluwer publishers, v.58, n. 3,p. 299-333, Marzo. 2005. 
BUENDÍA, G.; MONTIEL, G. From History to Research in Mathematics Education: socioepistemological elements for trigonometric function. In:KATZ, V. AND TZANAKIS, C. (Ed.).Recent Developments on Introducing a Historical Dimension in Mathematics Education. USA: Mathematical Association of America, 2011, p. 65-80.

CAMACHO, A. Socioepistemología y prácticas sociales. Revista Educación Matemática, México DF, v. 18, n. 1, p. 133-160, abr. 2006.

CANTORAL, R. Matemática Educativa. Un estudio de la formación social de la analiticidad. México DF: Grupo Editorial Iberoamérica SA de CV, 2001.

CANTORAL, R.; FARFÁN, R. Mathematics education: A vision of its evolution. Educational Studies in Mathematics, Kluwer publishers, v. 53, n. 3, p. 255-270, set. 2003.

CANTORAL, R.; FARFÁN, R.; LEZAMA, J.;MARTÍNEZ-SIERRA, G. Socioepistemología y representación: algunos ejemplos. Revista Latinoamericana de Matemática Educativa, México DF, p. 83-102, número especial 2006.

CORDERO, F. La distinción entre construcciones del cálculo. Una epistemología a través de la actividad humana. Revista Latinoamericana de Investigación en Matemática Educativa, México DF, v. 4, n. 2, p. 103-128, jul. 2001.

CORDERO, F.; FLORES, R. El uso de las gráficas en el discurso matemático escolar. Un estudio socioepistemológico en el nivel básico a través de los libros de texto. Revista Latinoamericana de Matemática Educativa, México, v. 10, n. 1, p. 7-38, mar. 2007.

COVIÁN, O. El papel del conocimiento matemático en la construcción de la vivienda tradicional. El caso de la cultura maya.2005. Tesis (Maestría en Matemática Educativa) - Centro de Investigación y de Estudios Avanzados, Instituto Politécnico Nacional, México D.F., 2005. (No publicada)

FAIRCLOUGH, N.; WODAK, R. Análisis crítico del discurso. In:VAN DIJK T. (Comp.) El discurso como interacción social. Estudios sobre el discurso II. Una integración multidisciplinaria.

Barcelona/Buenos Aires: Gedisa, 2001. Cap. 10, p. 867-404.

FLORES, R. (Ed.). Acta Latinoamericana de Matemática Educativa 25. México DF: Comité Latinoamericano de Matemática Educativa A.C., 2011.

GARCÍA-TORRES, E.; CANTORAL, R. Un estudio sobre los procesos de institucionalización de las prácticas en ingeniería biomédica. In: BUENDÍA, G.; MONTIEL, G. (Eds.) ESCUELA DE INVIERNO EN MATEMÁTICA EDUCATIVA, XI, 2007, Mérida, Yucatán. Memoria... México D.F.: Red de Centros de Investigación en Matemática Educativa, 2007, p. 483-494. Digitalizada.

GIOVANNINI, E. Matemática A para $6^{\circ}$ año. Funciones reales. Montevideo: Editorial Tradinco. $2001.405 \mathrm{p}$.

IAVA. Instituto “Alfredo Vásquez Acevedo". Algunas precisiones acerca del histórico edificio del Liceo N`35. Disponible en: http://iava.edu.uy/historia.htm. Acceso en: 14 ago. 2013.

LESTÓN, P. (Ed.).Acta Latinoamericana de Matemática Educativa 24. México DF: Comité Latinoamericano de Matemática Educativa A.C. 2011. 
MOLFINO, V. Procesos de institucionalización del concepto de límite: un análisis socioepistemológico. 2010. 217p. Tesis (doctorado en Matemática Educativa)- Centro de Investigación en Ciencia Aplicada y Tecnología Avanzada del IPN. (No publicada).

MOLFINO, V.;BUENDÍA, G. Análisis del Discurso como Acción Social: su rol en la construcción y difusión de conocimiento matemático. In:BUENDÍA G. (coord.). Reflexión e Investigación en Matemática Educativa. México DF: Lectorum, 2011a, p. 117-150.

MOLFINO, V. ;BUENDÍA, G. Análisis del discurso como acción social con relación al concepto de límite: un estudio de libros de texto. Premisa, Buenos Aires, v.13, n. 51, p. 3-15, nov. 2011 b.

MOLFINO, V.; BUENDÍA, G. El límite de funciones en la escuela: un análisis de su institucionalización. Revista electrónica de investigación en educación en ciencias, v.5, n. 1, p. 27 41, jul. 2010.

MONTIEL, G. Construcción de conocimiento trigonométrico. Un estudio socioepistemológico. México: Ediciones Díaz de Santos. 2011. 148 p.

REY PASTOR, J. Elementos de análisis algebraico. Madrid: Herederos de Julio Rey Pastor. 1917. $499 \mathrm{p}$.

REY PASTOR, J.; PI CALLEJA, P.; TREJO, C. Análisis matemático. Buenos Aires, Argentina: Kapelusz. 1952. 836 p.

TUYUB, I.; CANTORAL, R. Construcción social del conocimiento matemático durante la obtención de genes en una práctica toxicológica. Bolema, Rio Claro (SP), v. 26, n. 42A, p. 311-328, abr. 2012.

VAN DIJK, T. (Comp.).El discurso como interacción social. Una introducción multidisciplinaria. Barcelona/Buenos Aires: Gedisa. 2001. 464 p. 